\title{
The Association Between Guideline-concordant Care and Risk for Breast Cancer and Non-breast Cancer Mortality Among Older Women with Breast Cancer
}

\author{
Traci Le Masters ${ }^{1,2}$, Suresh Madhavan ${ }^{1,2,3}$, Usha Sambamoorthi ${ }^{1,2}$ \\ ${ }^{1}$ Department of Pharmaceutical Systems and Policy, School of Pharmacy, University, Morgantown, West Virginia, United States \\ ${ }^{2}$ West Virginia University Cancer Institute, West Virginia University, Morgantown, United States \\ ${ }^{3}$ College of Pharmacy, University of North Texas, Dallas-Fort Worth, United States
}

Email address:

tlemasters@hsc.wvu.edu (T. Le Masters)

\section{To cite this article:}

Traci Le Masters, Suresh Madhavan, Usha Sambamoorthi. The Association Between Guideline-concordant Care and Risk for Breast Cancer and Non-breast Cancer Mortality Among Older Women with Breast Cancer. Journal of Cancer Treatment and Research.

Vol. 7, No. 3, 2019, pp. 51-61. doi: 10.11648/j.jctr.20190703.12

Received: August 20, 2019; Accepted: September 6, 2019; Published: October 11, 2019

\begin{abstract}
The purpose of this study is to determine how receipt of guideline-concordant care (GCC) is associated with breast cancer-specific mortality (BCSM) and non-breast cancer mortality (NBCM) among older women with breast cancer. The SEER-Medicare data was used to identify 142, 433 women age $>66$ diagnosed with stage I-III breast cancer between 2007-2011. Receipt of GCC was determined according to evidence-based treatment guidelines. Cause-specific Cox proportional hazard multivariable regression models were used to estimate the association between GCC and the risk of BCSM, considering NBCM as a competing event, and NBCM, considering BCSM as a competing event, within five years of diagnosis or until end of follow-up. Among older women with breast cancer, $6.5 \%$ experienced BCSM and $11.9 \%$ experienced NBCM. GCC was associated with a $24 \%$ decreased risk of BCSM (AHR, 0.76; 95\% CI, 0.71-0.82), but a $80 \%$ increased risk of NBCM (AHR, 1.80; 95\% CI, 1.70-1.92). Receipt of adjuvant endocrine therapy was associated with an increased risk of BCSM and a decreased risk for NBCM. Receipt of chemotherapy was associated with an increased risk for BCSM and NBCM, while radiation therapy was associated with a decreased risk of NBCM. Women with a pre-existing dementia, arthritis, hypertension, stroke and increased comorbidity burden had an increased risk for BCSM. Most older breast cancer patients do not receive GCC, yet relatively few die from breast cancer. While GCC does decrease the risk of BCSM, the decision to treat should be made considering the patients existing health status, given that pre-existing comorbidity increases the risk for both BCSM and NBCM. Mortality differences associated with specific types of treatment may be attributed to patient selection for treatment based on worse cancer prognostic factors.
\end{abstract}

Keywords: Breast Cancer, Guideline-concordant Care, Survival

\section{Background}

Although the majority of older women diagnosed with breast cancer have less aggressive subtypes, [1, 2] older women experience worse breast cancer-specific mortality (BCSM) at every stage and sub-type, compared to younger women. [3] Older women with breast cancer are also at greater risk of non-breast cancer mortality (NBCM), especially those with greater comorbidity. [4] The concurrent increased risk for BCSM and NBCM may present challenges to the treatment decision making process for many older patients. While breast cancer treatment is primarily determined by evidence-based guidelines based on clinical characteristics and extent of disease spread, [5] other important considerations include patient preferences, health and age.

Yet, it is well documented that older breast cancer patients are often undertreated, as compared to their younger counterparts. [6, 7] In fact, a recent study reported that only $40 \%$ of women age $>66$ years received treatment according to evidence-based guidelines, or guidelineconcordant care (GCC), [8] possibly contributing to the 
worse BCSS observed among older women. Primary reasons associated with lower rates of GCC include older age, greater comorbidity, treatment toxicity, decreased functional status and limited life-expectancy. [9-11] Moreover, epidemiological studies have reported conflicting findings as to whether or not receipt of GCC and specific treatments are associated with improved BCSM and/or NBCM among older women. [12-14] An important, but previously unconsidered factor is the concept of competing risks of death. Competing events, such as NBCM, are important to account for when estimating cause-specific endpoints such as BCSM, [15] especially given that over $70 \%$ of deaths among women age $>75$ years with breast cancer, are due to non-breast cancer causes. [16] Therefore, the purpose of the current study is to investigate how GCC is associated with the risk of BCSM, considering $\mathrm{NBCM}$ as a competing event, and the risk of NBCM, considering BCSM as a competing event, among a large US population-based cohort of older women with breast cancer.

\section{Methods}

\subsection{Data Source and Cohort Definition}

The National Cancer Institute and Centers for Medicare and Medicaid Services collaborated to create the linked Surveillance, Epidemiology, and End Results (SEER)Medicare database. The SEER cancer registry, representing 17 distinct tumor registries and $26 \%$ of the US population, is linked to Medicare claims for individuals age $>65$ years using patient name, age, sex, date of birth, and social security number. [17] The linked database provides information regarding date of diagnosis, cancer site, stage, tumor characteristics, treatment, health conditions, health care use, patient enrollment and eligibility, selected demographic characteristics, and vital status information. For this study, the US Department of Health and Human Resource's 2009 Area Resource File (ARF) was additionally linked to the SEER-Medicare database to identify the area-level health resources using county and state identifiers. [18] Inclusion criteria for defining the study cohort included: female sex, age $>66$ years at the date of diagnosis, breast cancers diagnosed in the years 2007 - 2011, breast cancer was first and only cancer diagnosed during the study time period, diagnoses that were pathologically confirmed, patients who were alive for a minimum of 366 days after the date of diagnosis, stage of diagnosis was I, II or III, and continuous enrollment in Medicare Parts A and B fee-forservice plan 12 months before and after the date of diagnosis. Patients were excluded if they were diagnosed at death or autopsy, diagnosed at stage 0 or stage IV, enrolled in a health maintenance organization (HMO) plan at any time in the 12 months before and after diagnosis or tumor size was missing. The final analytic sample of 142,382 women.

\subsection{Measures}

\subsubsection{Outcomes}

The primary study outcome was BCSM, with NBCM treated as a competing event, and the secondary outcome was NBCM, with BCSM treated as a competing event. Causespecific mortality (BCSM) was ascertained using the SEER cause-specific death classification variable that determines cause of death by considering cause of death, sequence of tumor diagnosis, and site of primary tumor. This method reduces the risk for misclassification of the cause of death when using death certificate records. [19] All other causes of death were classified as NBCM. The follow-up period was for up to five years (1,830 days) after diagnosis or until the end of the study time-period (December $31^{\text {st }}, 2013$ ). Mortality events were identified using the Medicare date of death variable.

\subsubsection{Independent Variables}

The main independent variable was receipt of GCC (yes or no). Receipt of GCC was determined for each woman by comparing the actual course of treatment received to the recommended course of treatment, according to age and clinical characteristics as per National Comprehensive Cancer Network (NCCN) Breast Cancer Clinical Practice Guidelines. [20] Information regarding how GCC was determine has been described elsewhere. [8] Specific types of tests and treatments studied were receipt of estrogen receptor (ER) and progesterone receptor (PR) testing, breastconserving surgery (BCS), mastectomy, radiation therapy (RT), chemotherapy, initiation of chemotherapy within 120 days of diagnosis, and adjuvant hormone therapy (AET). Hormone receptor testing was estimated using a previously described method that considers documentation of a "positive", "negative", or "borderline" ER and PR status an indication that hormone receptor testing was conducted, and an "unknown" or "missing" status an indication that testing was not conducted. [21] Initiation of chemotherapy within 120 days of diagnosis, when indicated, was assessed according to joint American Society of Clinical Oncology (ASCO)/ NCCN quality measures. [22] These services were identified using International Classification of Diseases, 9th Revision (ICD-9) diagnostic and procedure codes and Healthcare Common Procedure Coding System (HCPCS)/Current Procedural Terminology (CPT) codes generic drug names (Table 1).

Table 1. Claims Codes Used for Identifying Types of Treatment.

\begin{tabular}{|c|c|c|c|c|c|}
\hline Type of Treatment & ICD-9 Diagnostic & ICD-9 Procedure & HCPCS/CPT & Revenue Center & Generic Drug Name \\
\hline $\begin{array}{l}\text { Breast-Conserving } \\
\text { Surgery }\end{array}$ & & $85.20-85.29$ & $\begin{array}{l}\text { 19120, 19125-19126, 19160, 19162, } \\
\text { 19301-19302 }\end{array}$ & & \\
\hline Mastectomy & & $85.33-85.36$ & $19140,19180,19182,19300,19303-$ & & \\
\hline
\end{tabular}




\begin{tabular}{|c|c|c|c|c|c|}
\hline Type of Treatment & ICD-9 Diagnostic & ICD-9 Procedure & HCPCS/CPT & Revenue Center & Generic Drug Name \\
\hline & & $85.40-85.48$ & $\begin{array}{l}\text { 19307, 19200, 19220, 19240, 19260, } \\
\text { 19271-19272 }\end{array}$ & & \\
\hline Radiation Therapy & $\begin{array}{l}\text { V58.0, V66.1, } \\
\text { V67.1 }\end{array}$ & $92.20-92.39$ & $\begin{array}{l}\text { 77261-77799, G0256, G0261, G0173- } \\
\text { G0174, G0243, G0251, G0338- } \\
\text { G03340 }\end{array}$ & 0330,0333 & \\
\hline Chemotherapy & $\begin{array}{l}\text { V58.1, V66.2, } \\
\text { V67.2, }\end{array}$ & $99.25,99.28$ & $\begin{array}{l}\text { 96400-96599, C8953-C8955, G0355- } \\
\text { G0363, G902-G9032, J0640, J8510, } \\
\text { J8520-J8521, J8530-J8999, J9000- } \\
\text { J9999, Q0083-Q0085, S9329-S9331 }\end{array}$ & $0331,0332,0335$ & \\
\hline $\begin{array}{l}\text { Adjuvant Endocrine } \\
\text { Therapy }\end{array}$ & & & & & $\begin{array}{l}\text { tamoxifen, } \\
\text { anastrozole, } \\
\text { exemestane, letrozle }\end{array}$ \\
\hline
\end{tabular}

Other independent variables were year of diagnosis, age, pre-existing chronic conditions, frequency of primary care provider (PCP) visits, clinical prognostic factors, oncology care resources, and demographic characteristics. Specific preexisting chronic conditions identified were anxiety, depression, dementia, arthritis, osteoporosis, diabetes, hypertension, hyperlipidemia, heart disease (includes coronary artery disease and cardiac arrhythmia), stroke, and chronic obstructive pulmonary disease (COPD), using methods described by the Multiple Chronic Conditions Working Group. [23] Comorbidity scores were calculated using the Klabunde adaptation of the Charlson Comorbidity Index (CCI) (score $=0$, 1, > 2). [24] Frequency of PCP visits was calculated by counting the number of unique PCP claim dates recorded one year before diagnosis in the physician claims file and dividing by the lower and upper 50th percent median cutoff (low, high). Clinical prognostic factors included stage at diagnosis, tumor size, lymph node status, hormone receptor status, and tumor grade. Measures of oncology care resources were the density of area-level mammography screening centers and oncology treatment centers relative to each woman's location of residence, using data from the ARF, categorized by the lower and upper 50th percent median cutoff (low, high). Surgeon specialty was assessed using provider specialty claims codes 02, 49 (general) and 83, 90, 91, 98 (oncology) from the physician claims file variable "hcfaspec" (general only, oncology only, both). Demographic characteristics included race, marital status, metro status, and 2010 Census measures of area-level education and annual income.

\subsection{Statistical Analysis}

Pearson $X^{2}$ tests were used to compare survival outcomes by patient characteristics. Cause-specific Cox proportional hazard multivariable regression models were used to estimate the risk of BCSM, with $\mathrm{NBCM}$ events treated as a censored observations, and the risk of NBCM, with BCSM events treated as a censored observations. These cause-specific regression models were adjusted for all other study variables. Independent variables, ER testing and PR testing, were removed from the final regression models due to small cell sizes. Sub-distribution hazard models were used to estimate the cumulative incidence functions for BCSM and NBCM, stratified by receipt of GCC (yes or no) using the Fine and Gray method. [25] Parameter estimates are presented as adjusted hazard ratios (AHR) with their corresponding 95\% confidence intervals $(\mathrm{CI}) . P$ values $<.05$ were considered statistically significant. All analyses were conducted using SAS version 9.4 software (SAS Institute Inc., Cary, NC). This study was approved for exemption by the West Virginia Institutional Review Board.

\section{Results}

\subsection{Unadjusted Analysis}

At follow-up, $6.5 \%$ of older women had experienced BCSM, $11.9 \%$ experienced NBCM and the majority did not receive GCC (Table 2). Among women who received GCC, a greater proportion were alive at follow-up (42.2\%), than experienced BCSM (33.2\%) or NBCM (26.1\%). Whereas, greater proportions of women who did not receive $\mathrm{GCC}$ experienced BCSM $(66.8 \%)$ or NBCM $(73.9 \%)$, than living at follow-up $(57.8 \%)$. Greater proportions of women who were hormone receptor negative experienced BCSM $(27.5 \%)$, than NBCM (9.2\%) or were alive (10.2\%). Among those who received chemotherapy, a greater proportion experienced BCSM (46.1\%), than NBCM (16.9\%) or were alive $(28.8 \%)$. Greater proportions of women who received AET or RT were still alive at follow-up, than experienced BCSM or NBCM.

Table 2. Comparison of Characteristics among Older Women Diagnosed with Breast Cancer by 5-Year Survival Outcomes.

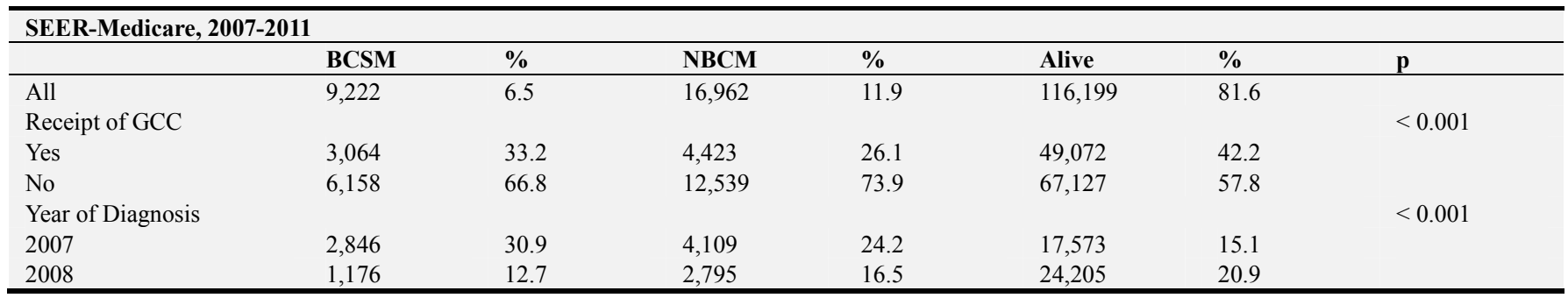




\begin{tabular}{|c|c|c|c|c|c|c|c|}
\hline \multicolumn{8}{|l|}{ SEER-Medicare, 2007-2011 } \\
\hline & BCSM & $\%$ & NBCM & $\%$ & Alive & $\%$ & p \\
\hline 2009 & 3,569 & 38.7 & 3,595 & 21.2 & 24,196 & 20.8 & \\
\hline 2010 & 1,109 & 12.0 & 4,571 & 27.0 & 24,641 & 21.2 & \\
\hline 2011 & 522 & 5.7 & 1,892 & 11.1 & 25,584 & 22.0 & \\
\hline \multicolumn{8}{|l|}{ Age \& Health } \\
\hline Age at Diagnosis & & & & & & & $<0.001$ \\
\hline $66-69$ & 1,942 & 21.1 & 719 & 4.2 & 26,344 & 22.7 & \\
\hline $70-74$ & 1,548 & 16.8 & 1,579 & 9.3 & 32,904 & 28.3 & \\
\hline $75-79$ & 2,010 & 21.8 & 3,950 & 23.3 & 27,098 & 23.3 & \\
\hline$>80$ & 3,722 & 40.3 & 10,714 & 63.2 & 29,853 & 25.7 & \\
\hline PCP Visits & & & & & & & $<0.001$ \\
\hline Low & 3,963 & 43.0 & 6,599 & 38.9 & 54,042 & 46.5 & \\
\hline High & 5,259 & 57.0 & 10,363 & 61.1 & 62,157 & 53.5 & \\
\hline Anxiety & & & & & & & $<0.001$ \\
\hline Yes & 259 & 2.8 & 2,611 & 15.4 & 9,131 & 7.9 & \\
\hline No & 8,963 & 97.2 & 14,351 & 84.6 & 10,7068 & 92.1 & \\
\hline Depression & & & & & & & $<0.001$ \\
\hline Yes & 773 & 8.4 & 2,584 & 15.2 & 9,021 & 7.8 & \\
\hline No & 8,449 & 91.6 & 14,378 & 84.8 & 107,178 & 92.2 & \\
\hline Dementia & & & & & & & $<0.001$ \\
\hline Yes & 1,369 & 14.8 & 3,849 & 22.7 & 2,605 & 2.2 & \\
\hline No & 7,853 & 85.2 & 13,113 & 77.3 & 113,594 & 97.8 & \\
\hline Arthritis & & & & & & & $<0.001$ \\
\hline Yes & 3,666 & 39.8 & 4,093 & 24.1 & 32,614 & 28.1 & \\
\hline No & 5,556 & 60.2 & 12,869 & 75.9 & 83,585 & 71.9 & \\
\hline Osteoporosis & & & & & & & $<0.001$ \\
\hline Yes & 939 & 10.2 & 1,326 & 7.8 & 14,336 & 12.3 & \\
\hline No & 8,283 & 89.8 & 15,636 & 92.2 & 101,863 & 87.7 & \\
\hline Diabetes & & & & & & & $<0.001$ \\
\hline Yes & 3,289 & 35.7 & 7,336 & 43.2 & 35,528 & 30.6 & \\
\hline No & 5,933 & 64.3 & 9,626 & 56.8 & 80,671 & 69.4 & \\
\hline Hypertension & & & & & & & $<0.001$ \\
\hline Yes & 7,965 & 86.4 & 14,791 & 87.2 & 93,471 & 80.4 & \\
\hline No & 1,257 & 13.6 & 2,171 & 12.8 & 22,728 & 19.6 & \\
\hline Hyperlipidemia & & & & & & & $<0.001$ \\
\hline Yes & 5,218 & 56.6 & 10,498 & 61.9 & 77,844 & 67.0 & \\
\hline No & 4,004 & 43.4 & 6,464 & 38.1 & 38,355 & 33.0 & \\
\hline \multicolumn{8}{|l|}{ Heart Disease } \\
\hline Yes & 3,991 & 43.3 & 9,420 & 55.5 & 38,536 & 33.2 & \\
\hline No & 5,231 & 56.7 & 7,542 & 44.5 & 77,663 & 66.8 & \\
\hline Stroke & & & & & & & $<0.001$ \\
\hline Yes & 1,035 & 11.2 & 3,275 & 19.3 & 6,701 & 5.8 & \\
\hline No & 8,187 & 88.8 & 13,687 & 80.7 & 109,498 & 94.2 & \\
\hline COPD & & & & & & & $<0.001$ \\
\hline Yes & 1,547 & 16.8 & 4,158 & 24.5 & 11,636 & 10.0 & \\
\hline No & 7,675 & 83.2 & 12,804 & 75.5 & 104,563 & 90.0 & \\
\hline Charlson Comorbidity Index & & & & & & & $<0.001$ \\
\hline 0 & 4,540 & 49.2 & 7,281 & 42.9 & 71,484 & 61.5 & \\
\hline 1 & 2,982 & 32.4 & 5,106 & 30.1 & 31,946 & 27.5 & \\
\hline$>2$ & 1,700 & 18.4 & 4,575 & 27.0 & 12,769 & 11.0 & \\
\hline \multicolumn{8}{|l|}{ Clinical Prognostic Factors } \\
\hline Stage at Diagnosis & & & & & & & $<0.001$ \\
\hline I & 1,178 & 12.8 & 7,643 & 45.1 & 67,350 & 58.0 & \\
\hline II & 4,638 & 50.3 & 7,328 & 43.2 & 39,743 & 34.2 & \\
\hline III & 3,406 & 36.9 & 1,991 & 11.7 & 9,106 & 7.8 & \\
\hline Tumor Size & & & & & & & $<0.001$ \\
\hline$<1 \mathrm{~cm}$ & 527 & 5.7 & 2,670 & 15.7 & 32,843 & 28.3 & \\
\hline$<2 \mathrm{~cm}$ & 1,981 & 21.5 & 6,461 & 38.1 & 47,584 & 40.9 & \\
\hline $2-5 \mathrm{~cm}$ & 4,615 & 50.0 & 6,904 & 40.7 & 33,238 & 28.6 & \\
\hline$>5 \mathrm{~cm}$ & 2,099 & 22.8 & 927 & 5.5 & 2,534 & 2.2 & \\
\hline Lymph Nodes & & & & & & & $<0.001$ \\
\hline Positive & 4,297 & 46.6 & 3,903 & 23.0 & 28,450 & 24.5 & \\
\hline Negative & 4,925 & 53.4 & 13,059 & 77.0 & 87,749 & 75.5 & \\
\hline Hormone Receptor Status & & & & & & & $<0.001$ \\
\hline ER and/or PR Positive & 5,395 & 58.5 & 14,811 & 87.3 & 99,525 & 85.6 & \\
\hline ER and PR Negative & 2,538 & 27.5 & 1,561 & 9.2 & 11,812 & 10.2 & \\
\hline Borderline/Unknown & 1,289 & 14.0 & 590 & 3.5 & 4,862 & 4.2 & \\
\hline
\end{tabular}




\begin{tabular}{|c|c|c|c|c|c|c|c|}
\hline \multicolumn{8}{|l|}{ SEER-Medicare, 2007-2011 } \\
\hline & BCSM & $\%$ & NBCM & $\%$ & Alive & $\%$ & $\mathbf{p}$ \\
\hline \multicolumn{7}{|l|}{ Tumor Grade } & \multirow[t]{5}{*}{$<0.001$} \\
\hline Well Differentiated & 378 & 4.1 & 3,684 & 21.7 & 28,519 & 24.5 & \\
\hline Moderately Differentiated & 2,661 & 28.8 & 8,226 & 48.5 & 52,016 & 44.8 & \\
\hline Poorly Differentiated & 4,940 & 53.6 & 4,057 & 23.9 & 28,341 & 24.4 & \\
\hline Undifferentiated/Unknown & 1,243 & 13.5 & 995 & 5.9 & 7,323 & 6.3 & \\
\hline \multicolumn{8}{|l|}{ Oncology Care Resources } \\
\hline \multicolumn{7}{|c|}{ Mammography Screening Centers } & \multirow[t]{3}{*}{$<0.001$} \\
\hline Low & 3,019 & 32.7 & 8,658 & 51.0 & 63,966 & 55.1 & \\
\hline High & 6,203 & 67.3 & 8,304 & 49.0 & 52,233 & 44.9 & \\
\hline \multicolumn{7}{|l|}{ Oncology Treatment Centers } & \multirow[t]{3}{*}{$<0.001$} \\
\hline Low & 3,051 & 33.1 & 8,740 & 51.5 & 64,563 & 55.6 & \\
\hline High & 6,171 & 66.9 & 8,222 & 48.5 & 51,636 & 44.4 & \\
\hline \multicolumn{7}{|c|}{ Specialty of Treating Surgeon (s) } & \multirow[t]{4}{*}{$<0.001$} \\
\hline General Only & 1,305 & 14.2 & 4,273 & 25.2 & 13,450 & 11.6 & \\
\hline Oncology Only & 407 & 4.4 & 933 & 5.5 & 7,580 & 6.5 & \\
\hline Both & 7,510 & 81.4 & 11,756 & 69.3 & 95,169 & 81.9 & \\
\hline \multicolumn{8}{|c|}{ Demographic Characteristics } \\
\hline \multicolumn{7}{|l|}{ Race/Ethnicity } & \multirow[t]{5}{*}{$<0.001$} \\
\hline White & 6,092 & 66.1 & 12,440 & 73.3 & 88,254 & 75.9 & \\
\hline Black & 2,814 & 30.5 & 4,064 & 24.0 & 20,202 & 17.4 & \\
\hline Hispanic/Latino & 231 & 2.5 & 178 & 1.0 & 4,406 & 3.8 & \\
\hline Other & 85 & 0.9 & 280 & 1.7 & 3,337 & 2.9 & \\
\hline \multicolumn{7}{|l|}{ Education } & \multirow[t]{3}{*}{$<0.001$} \\
\hline$<15 \%$ college degree & 3,681 & 39.9 & 6,320 & 37.3 & 44,272 & 38.1 & \\
\hline$>15 \%$ college degree & 5,541 & 60.1 & 10,642 & 62.7 & 71,927 & 61.9 & \\
\hline Annual Income & & & & & & & 0.003 \\
\hline$<\$ 35,000$ & 3,381 & 36.7 & 5,951 & 35.1 & 40,535 & 34.9 & \\
\hline$>\$ 35,000$ & 5,841 & 63.3 & 11,011 & 64.9 & 75,664 & 65.1 & \\
\hline Marital Status & & & & & & & $<0.001$ \\
\hline Yes Married/Partnered & 976 & 10.6 & 4,642 & 27.4 & 39,531 & 34.0 & \\
\hline No not Married/Partnered & 8,246 & 89.4 & 12,320 & 72.6 & 76,668 & 66.0 & \\
\hline Metro Status & & & & & & & $<0.001$ \\
\hline Non-metro & 308 & 3.3 & 1,774 & 10.5 & 7,315 & 6.3 & \\
\hline Metro & 8,914 & 96.7 & 15,188 & 89.5 & 108,884 & 93.7 & \\
\hline Tests \& Treatments & & & & & & & \\
\hline ER Status Tested & & & & & & & $<0.001$ \\
\hline Yes & 8,291 & 89.9 & 16,371 & 96.5 & 111,334 & 95.8 & \\
\hline No & 931 & 10.1 & 591 & 3.5 & 4,865 & 4.2 & \\
\hline PR Status Tested & & & & & & & $<0.001$ \\
\hline Yes & 8,291 & 89.9 & 16,365 & 96.5 & 111,005 & 95.5 & \\
\hline No & 931 & 10.1 & 597 & 3.5 & 5,194 & 4.5 & \\
\hline Received AET & & & & & & & $<0.001$ \\
\hline Yes & 3,717 & 40.3 & 7,000 & 41.3 & 59,989 & 51.6 & \\
\hline No & 5,505 & 59.7 & 9,962 & 58.7 & 56,210 & 48.4 & \\
\hline Type of Surgery & & & & & & & $<0.001$ \\
\hline BCS & 3,930 & 42.6 & 10,069 & 59.4 & 72,546 & 62.4 & \\
\hline Mastectomy & 2,553 & 27.7 & 3,105 & 18.3 & 23,258 & 20.0 & \\
\hline BCS and Mastectomy & 1,524 & 16.5 & 2,878 & 17.0 & 17,439 & 15.0 & \\
\hline No Surgery & 1,215 & 13.2 & 910 & 5.3 & 2,956 & 2.6 & \\
\hline Received Radiation Therapy & & & & & & & $<0.001$ \\
\hline Yes & 4,757 & 51.6 & 5,708 & 33.7 & 71,275 & 61.3 & \\
\hline No & 4,465 & 48.4 & 11,254 & 66.3 & 44,924 & 38.7 & \\
\hline Received Chemotherapy & & & & & & & $<0.001$ \\
\hline Yes & 4,253 & 46.1 & 2,862 & 16.9 & 33,442 & 28.8 & \\
\hline No & 4,969 & 53.9 & 14,100 & 83.1 & 82,757 & 71.1 & \\
\hline Time to Chemotherapy & & & & & & & $<0.001$ \\
\hline Appropriate & 2,819 & 30.6 & 1,520 & 9.0 & 23,812 & 20.5 & \\
\hline Not Appropriate & 1,434 & 15.5 & 1,342 & 7.9 & 9,630 & 8.3 & \\
\hline No Chemotherapy & 4,969 & 53.9 & 14,100 & 83.1 & 82,757 & 71.2 & \\
\hline
\end{tabular}

Abbreviations: GCC, guideline-concordant care; BCSM, breast cancer specific mortality; NBCM, non-breast cancer mortality; PCP, primary care physician; $\mathrm{ER}$, estrogen receptor; PR, progesterone receptor; AET, adjuvant endocrine therapy; COPD, chronic obstructive pulmonary disease.

\subsection{Breast Cancer-Specific Mortality}

In the multivariate analysis, women who received GCC had a $24 \%$ lower risk of BCSM (AHR, 0.76; 95\% CI, 0.71$0.82)$, than those who did not receive GCC (Table 3). Women with a CCI > $2($ AHR, 3.10; $95 \% \mathrm{CI}, 2.81-3.43)$ or with 
dementia (AHR, 3.57; 95\% CI, 3.30-3.87) were more than three times as likely to experience BCSM. Those with arthritis, hypertension and stroke also had a higher risk for BCSM. Women who were lymph node positive had a decreased risk of BCSM (AHR, 0.58; 95\% CI, 0.54- 0.62), while those who were later stage, with larger tumors, hormone receptor negative or with moderately and poorly differentiated tumors had increased risk for BCSM. Women who received AET had a $59 \%$ increased risk of BCSM
(AHR, 1.59; 95\% CI, 1.50-1.68). Receipt of chemotherapy was also associated with an increased risk for BCSM (AHR, 1.18; 95\% CI, 1.08-1.29). Women of black race, Hispanic/Latino ethnicity, and other race had a decreased risk of BCSM, compared to white women. At five years (1830 days), the cumulative incidence of BCSM was significantly greater among women who did not receive GCC [(HR, 0.054; 95\% CI, 0.052-0.056); Gray's test $p<0.0001$ ] (Figure 1).

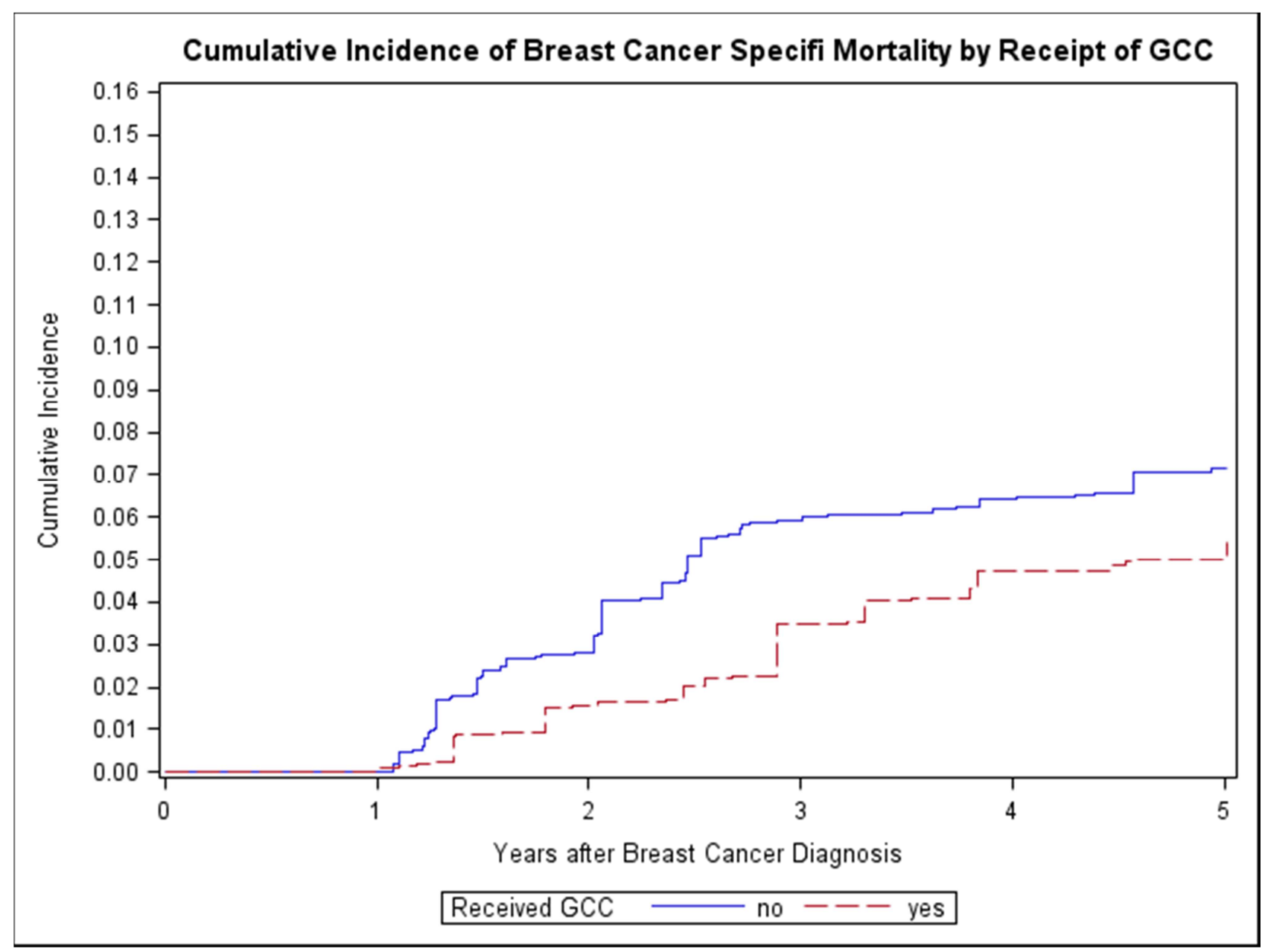

Figure 1. Competing Risks Cumulative Incidence of Breast Cancer Specific Mortality.

Table 3. Adjusted Cause-Specific Cox Proportional Hazard Models for Older Women Diagnosed with Breast Cancer.

\begin{tabular}{|c|c|c|c|c|c|c|}
\hline \multicolumn{7}{|c|}{ SEER-Medicare, 2007-2011 } \\
\hline & \multicolumn{3}{|c|}{ BCSM vs. Alive } & \multicolumn{3}{|c|}{ NBCM vs. Alive } \\
\hline & AHR & $95 \%$ CI & Sig. & AHR & $95 \% \mathrm{CI}$ & Sig. \\
\hline \multicolumn{7}{|c|}{ Adjusted Hazard Ratio } \\
\hline \multicolumn{7}{|c|}{ Receipt of GCC } \\
\hline Yes & 0.76 & {$[0.71,0.82]$} & $* * *$ & 1.80 & {$[1.70,1.92]$} & $* * *$ \\
\hline No & 1.00 & - & & 1.00 & - & \\
\hline \multicolumn{7}{|c|}{ Year of Diagnosis } \\
\hline 2007 & 1.00 & - & & 1.00 & - & \\
\hline 2008 & 0.42 & {$[0.39,0.46]$} & $* * *$ & 0.77 & {$[0.73,0.81]$} & *** \\
\hline 2009 & 1.17 & {$[1.10,1.24]$} & $* * *$ & 0.69 & {$[0.66,0.73]$} & $* * *$ \\
\hline 2010 & 0.14 & {$[0.13,0.16]$} & $* * *$ & 0.98 & {$[0.94,1.03]$} & \\
\hline 2011 & 0.18 & {$[0.16,0.20]$} & $* * *$ & 0.38 & {$[0.35,0.40]$} & *** \\
\hline \multicolumn{7}{|c|}{ Age \& Health } \\
\hline \multicolumn{7}{|c|}{ Age at Diagnosis } \\
\hline $66-69$ & 1.00 & 一 & & 1.00 & - & \\
\hline
\end{tabular}




\begin{tabular}{|c|c|c|c|c|c|c|}
\hline \multicolumn{7}{|c|}{ SEER-Medicare, 2007-2011 } \\
\hline & \multicolumn{3}{|c|}{ BCSM vs. Alive } & \multicolumn{3}{|c|}{ NBCM vs. Alive } \\
\hline & AHR & $95 \%$ CI & Sig. & AHR & $95 \%$ CI & Sig. \\
\hline $70-74$ & 0.71 & {$[0.66,0.77]$} & $* * *$ & 1.53 & {$[1.40,1.68]$} & \\
\hline 75-79 & 1.39 & {$[1.29,1.50]$} & $* * *$ & 4.59 & {$[4.59,5.40]$} & $* * *$ \\
\hline$>80$ & 1.27 & {$[1.29,1.50]$} & $* * *$ & 7.79 & {$[7.18,8.45]$} & $* * *$ \\
\hline \multicolumn{7}{|l|}{ PCP Visits } \\
\hline Low & 1.00 & - & & 1.00 & - & \\
\hline High & 1.09 & {$[1.03,1.14]$} & $* * *$ & 0.84 & {$[0.81,0.87]$} & $* * *$ \\
\hline \multicolumn{7}{|l|}{ Anxiety } \\
\hline Yes & 0.26 & {$[0.23,0.30]$} & $* * *$ & 1.65 & {$[1.57,1.74]$} & $* * *$ \\
\hline No & 1.00 & - & & 1.00 & - & \\
\hline \multicolumn{7}{|l|}{ Depression } \\
\hline Yes & 0.87 & {$[0.80,0.96]$} & $* *$ & 1.20 & {$[1.14,1.26]$} & $* * *$ \\
\hline No & 1.00 & - & & 1.00 & - & \\
\hline \multicolumn{7}{|l|}{ Dementia } \\
\hline Yes & 3.67 & {$[3.39,3.97]$} & $* * *$ & 3.11 & {$[2.96,3.27]$} & $* * *$ \\
\hline No & 1.00 & - & & 1.00 & - & \\
\hline \multicolumn{7}{|l|}{ Arthritis } \\
\hline Yes & 1.25 & {$[1.19,1.31]$} & $* * *$ & 0.61 & {$[0.59,0.64]$} & $* * *$ \\
\hline No & 1.00 & - & & 1.00 & - & \\
\hline \multicolumn{7}{|l|}{ Osteoporosis } \\
\hline Yes & 0.83 & {$[0.77,0.90]$} & $* * *$ & 0.55 & {$[0.52,0.59]$} & $* * *$ \\
\hline No & 1.00 & - & & 1.00 & - & \\
\hline \multicolumn{7}{|l|}{ Diabetes } \\
\hline Yes & 0.64 & {$[0.59,0.69]$} & $* * *$ & 1.58 & {$[1.51,1.66]$} & $* * *$ \\
\hline No & 1.00 & - & & 1.00 & - & \\
\hline \multicolumn{7}{|l|}{ Hypertension } \\
\hline Yes & 1.53 & {$[1.42,1.64]$} & $* * *$ & 1.09 & {$[1.03,1.14]$} & $* *$ \\
\hline No & 1.00 & - & & 1.00 & - & \\
\hline \multicolumn{7}{|l|}{ Hyperlipidemia } \\
\hline Yes & 0.63 & {$[0.60,0.66]$} & $* * *$ & 0.95 & {$[0.92,0.99]$} & $* *$ \\
\hline No & 1.00 & - & & 1.00 & - & \\
\hline \multicolumn{7}{|l|}{ Heart Disease } \\
\hline Yes & 1.11 & {$[1.05,1.16]$} & $* * *$ & 1.35 & {$[1.31,1.41]$} & $* * *$ \\
\hline No & 1.00 & - & & 1.00 & - & \\
\hline Stroke & & & & & & \\
\hline Yes & 1.89 & {$[1.76,2.04]$} & $* * *$ & 1.70 & {$[1.63,1.78]$} & $* * *$ \\
\hline No & 1.00 & - & & 1.00 & - & \\
\hline COPD & & & & & & \\
\hline Yes & 0.96 & {$[0.89,1.03]$} & & 2.54 & {$[2.42,2.67]$} & $* * *$ \\
\hline No & 1.00 & - & & 1.00 & - & \\
\hline Charlson Comorbidity & & & & & & \\
\hline 0 & 1.00 & - & & 1.00 & - & \\
\hline 1 & 1.86 & {$[1.73,2.00]$} & $* * *$ & 0.69 & {$[0.65,0.73]$} & $* * *$ \\
\hline$>2$ & 3.10 & {$[2.81,3.43]$} & $* * *$ & 1.13 & {$[1.13,1.28]$} & $* * *$ \\
\hline Clinical Prognostic Fac & & & & & & \\
\hline Stage at Diagnosis & & & & & & \\
\hline I & 1.00 & - & & 1.00 & - & \\
\hline II & 3.81 & {$[3.42,4.24]$} & $* * *$ & 1.43 & {$[1.32,1.54]$} & $* * *$ \\
\hline III & 15.39 & {$[13.57,17.44]$} & $* * *$ & 1.78 & {$[1.61,1.96]$} & $* * *$ \\
\hline Tumor Size & & & & & & \\
\hline$<1 \mathrm{~cm}$ & 1.00 & - & & 1.00 & - & \\
\hline$<2 \mathrm{~cm}$ & 1.36 & {$[1.23,1.50]$} & $* * *$ & 1.34 & {$[1.28,1.41]$} & $* * *$ \\
\hline $2-5 \mathrm{~cm}$ & 1.33 & {$[1.18,1.49]$} & $* * *$ & 1.15 & {$[1.06,1.25]$} & $* * *$ \\
\hline$>5 \mathrm{~cm}$ & 4.29 & {$[3.79,4.86]$} & $* * *$ & 2.15 & {$[1.93,2.39]$} & $* * *$ \\
\hline Lymph Nodes & & & & & & \\
\hline Positive & 0.58 & {$[0.54,0.62]$} & $* * *$ & 1.09 & {$[1.03,1.16]$} & $* *$ \\
\hline Negative & 1.00 & - & & 1.00 & - & \\
\hline Hormone Receptor Stat & & & & & & \\
\hline ER and/or PR Positive & 1.00 & - & & 1.00 & - & \\
\hline ER and PR Negative & 3.75 & {$[3.50,4.01]$} & $* * *$ & 0.71 & {$[0.67,0.76]$} & $* * *$ \\
\hline Borderline/Unknown & 2.37 & {$[2.18,2.57]$} & $* * *$ & 0.71 & {$[0.65,0.78]$} & $* * *$ \\
\hline Tumor Grade & & & & & & \\
\hline Well Differentiated & 1.00 & - & & 1.00 & - & \\
\hline
\end{tabular}




\begin{tabular}{|c|c|c|c|c|c|c|}
\hline \multicolumn{7}{|l|}{ SEER-Medicare, 2007-2011 } \\
\hline & \multicolumn{3}{|c|}{ BCSM vs. Alive } & \multicolumn{3}{|c|}{ NBCM vs. Alive } \\
\hline & AHR & $95 \% \mathrm{CI}$ & Sig. & AHR & $95 \%$ CI & Sig. \\
\hline Moderately Differentiated & 2.46 & {$[2.18,2.78]$} & $* * *$ & 0.95 & {$[0.91,0.99]$} & $*$ \\
\hline Poorly Differentiated & 4.85 & {$[4.29,5.48]$} & $* * *$ & 1.03 & {$[0.98,1.09]$} & \\
\hline Undifferentiated/Unknown & 4.80 & {$[4.20,5.48]$} & $* * *$ & 0.81 & {$[0.75,0.87]$} & $* * *$ \\
\hline \multicolumn{7}{|l|}{ Oncology Care Resources } \\
\hline \multicolumn{7}{|c|}{ Mammography Screening Centers } \\
\hline Low & 1.00 & - & & 1.00 & - & \\
\hline High & 1.01 & {$[0.74,1.38]$} & & 1.14 & {$[0.96,1.34]$} & \\
\hline \multicolumn{7}{|l|}{ Oncology Treatment Centers } \\
\hline Low & 1.00 & - & & 1.00 & - & \\
\hline High & 2.23 & {$[1.63,3.03]$} & $* * *$ & 1.10 & {$[0.96,1.34]$} & \\
\hline \multicolumn{7}{|c|}{ Specialty of Treating Surgeon (s) } \\
\hline General Only & 1.00 & - & & 1.00 & - & \\
\hline Oncology Only & 0.53 & {$[0.46,0.60]$} & $* * *$ & 1.07 & {$[0.99,1.16]$} & \\
\hline Both & 1.08 & {$[1.01,1.16]$} & $*$ & 1.03 & {$[0.99,1.08]$} & \\
\hline \multicolumn{7}{|c|}{ Socio-Demographic Characteristics } \\
\hline \multicolumn{7}{|l|}{ Race } \\
\hline White & 1.00 & - & & 1.00 & - & \\
\hline Black & 0.65 & {$[0.61,0.69]$} & $* * *$ & 0.92 & {$[0.88,0.96]$} & $* * *$ \\
\hline Hispanic/Latino & 0.69 & {$[0.59,0.77]$} & $* * *$ & 0.38 & {$[0.32,0.44]$} & $* * *$ \\
\hline Other & 0.73 & {$[0.58,0.92]$} & $* * *$ & 0.87 & {$[0.77,0.98]$} & $*$ \\
\hline \multicolumn{7}{|l|}{ Education } \\
\hline$<15 \%$ college degree & 1.00 & - & & 1.00 & - & \\
\hline$>15 \%$ college degree & 0.96 & {$[0.91,1.00]$} & & 1.05 & {$[1.01,1.09]$} & $*$ \\
\hline \multicolumn{7}{|l|}{ nnual Income } \\
\hline$<\$ 35,000$ & 1.00 & - & & 1.00 & - & \\
\hline$>\$ 35,000$ & 0.96 & {$[0.92,1.01]$} & & 0.98 & {$[0.95,1.02]$} & \\
\hline \multicolumn{7}{|l|}{ Marital Status } \\
\hline Yes Married/Partnered & 1.00 & - & & 1.00 & - & \\
\hline No not Married/Partnered & 3.24 & {$[3.01,3.49]$} & $* * *$ & 0.90 & {$[0.86,0.93]$} & $* * *$ \\
\hline \multicolumn{7}{|l|}{ Metro Status } \\
\hline Non-metro & 2.71 & {$[2.32,3.29]$} & $* * *$ & 1.48 & {$[1.36,1.60]$} & $* * *$ \\
\hline Metro & 1.00 & - & & 1.00 & - & \\
\hline \multicolumn{7}{|l|}{ Tests \& Treatments } \\
\hline \multicolumn{7}{|l|}{ Received AET } \\
\hline Yes & 1.59 & {$[1.49,1.68]$} & $* * *$ & 0.57 & {$[0.54,0.59]$} & $* * *$ \\
\hline No & 1.00 & - & & 1.00 & - & \\
\hline \multicolumn{7}{|l|}{ Type of Surgery } \\
\hline BCS & 1.00 & - & & 1.00 & - & \\
\hline Mastectomy & 0.89 & {$[0.83,0.96]$} & $* * *$ & 0.54 & {$[0.51,0.57]$} & $* * *$ \\
\hline BCS and Mastectomy & 0.72 & {$[0.67,0.78]$} & $* * *$ & 1.11 & {$[1.06,1.17]$} & $* * *$ \\
\hline No Surgery & 1.42 & {$[1.29,1.56]$} & $* * *$ & 0.84 & {$[0.77,0.91]$} & $* * *$ \\
\hline \multicolumn{7}{|l|}{ Received Radiation Therapy } \\
\hline Yes & 1.03 & {$[0.97,1.10]$} & & 0.43 & {$[0.41,0.46]$} & $* * *$ \\
\hline No & 1.00 & - & & 1.00 & - & \\
\hline \multicolumn{7}{|l|}{ Received Chemotherapy } \\
\hline Yes & 1.18 & {$[1.08,1.29]$} & $* * *$ & 1.48 & {$[1.38,1.58]$} & $* * *$ \\
\hline No & 1.00 & - & & 1.00 & - & \\
\hline \multicolumn{7}{|l|}{ ime to Chemotherapy } \\
\hline Appropriate & 1.51 & {$[1.37,1.66]$} & $* * *$ & 0.45 & {$[0.41,0.48]$} & $* * *$ \\
\hline Not Appropriate & 1.00 & - & & 1.00 & - & \\
\hline No Chemotherapy & - & - & & - & - & \\
\hline
\end{tabular}

$*=p<0.05 ; * *=p<0.01 ; * * *=p<0.001$; Abbreviations: AHR, adjusted hazard ratio; BCSM, breast cancer specific mortality; NBCM, non-breast cancer mortality; PCP, primary care physician; ER, estrogen receptor; PR, progesterone receptor; AET, adjuvant endocrine therapy; COPD, chronic obstructive pulmonary disease.

\subsection{Non-Breast Cancer Mortality}

Women who received GCC had an $80 \%$ increased risk for NBCM (AHR, 1.80; 95\% CI, 1.70-1.92), compared to those who did not receive GCC. Women who received AET (AHR, 0.57 ; $95 \% \mathrm{CI}, 0.54-0.59$ ) or RT (AHR, 0.43 ; 95\% CI, 0.41 -
0.46) had a decreased risk for NBCM, while women who received chemotherapy had a $48 \%$ increased risk for NBCM (AHR, 1.48; 95\% CI, 1.38-1.58). The five-year cumulative incidence of NBCM was significantly greater for those who did not receive GCC [(HR, 0.078; 95\% CI, 0.076-0.080); Gray's test $p<0.0001$ ] (Figure 2). 


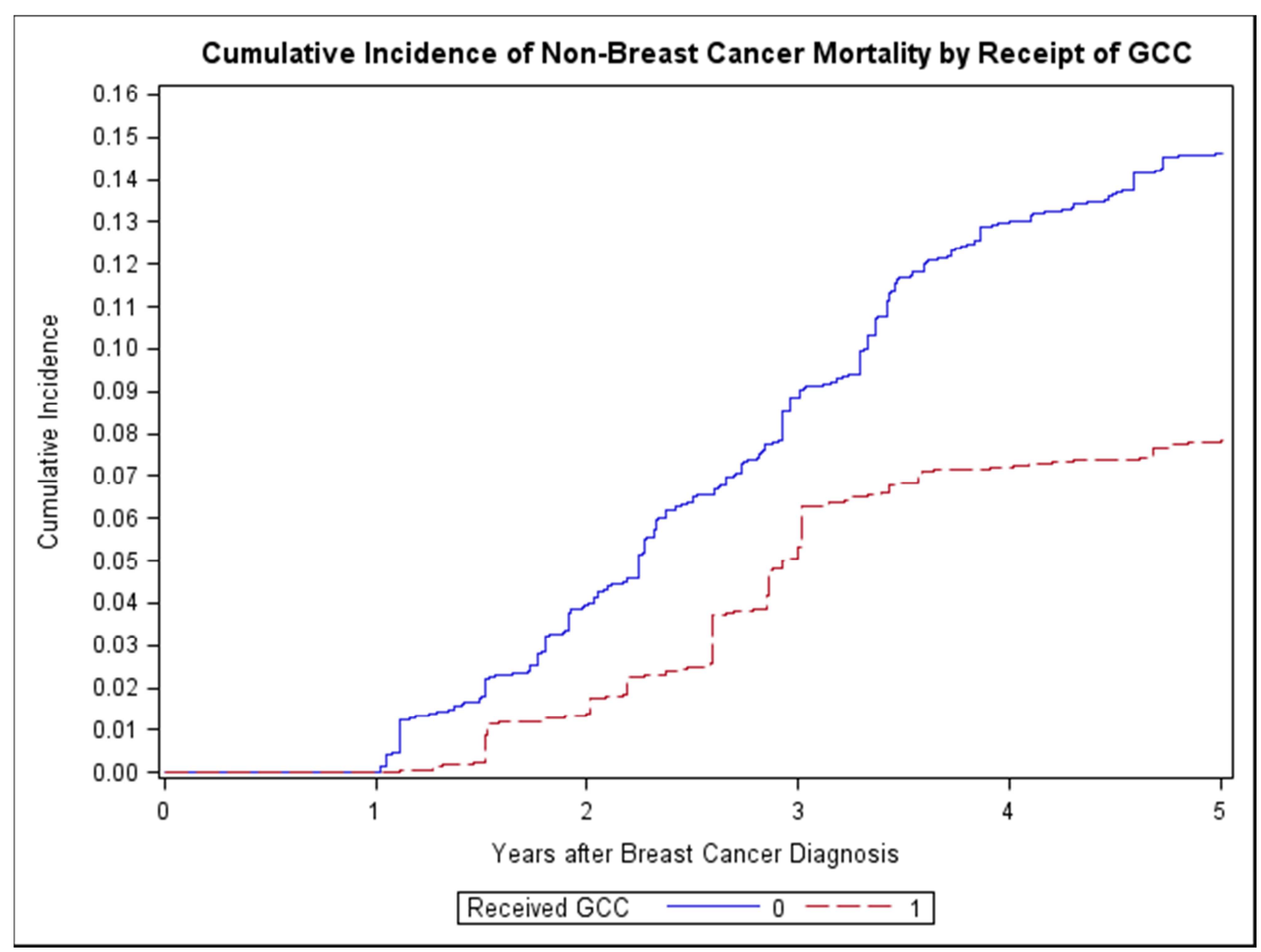

Figure 2. Competing Risks Cumulative Incidence Non-Breast Cancer Mortality.

\section{Discussion}

Although undertreatment has been well document among older women with breast cancer, the competing risks of BCSM and NBCM may complicate breast cancer treatment decisions and create uncertainty regarding the benefits and harms of GCC for this patient population. Therefore, this study sought to determine how receipt of GCC is associated with the risk of BCSM, considering NBCM as a competing event, and NBCM, considering BCSM as a competing event. Among 142,382 elderly women with stage I - III breast cancer, the majority did not receive GCC, but only $6.5 \%$ experienced BCSM. Yet, women who did receive GCC had a $22 \%$ decreased risk for BCSM, but a $69 \%$ increased risk for NBCM. The difference in the associated risk between GCC and BCSM and NBCM, may be explained by the trade-off of benefits and harms of specific types of treatment. In addition to increased intolerance of sideeffects that include nausea, vomiting, diarrhea, neutropenia, and fatigue, chemotherapeutic agents, especially anthracyclinebased agents, have a known risk for cardiotoxic and hepatotoxic effects, $[10,11]$ in older patients with geriatric syndromes, pre-existing chronic conditions, or impaired organ function, and increased risk for treatment-related mortality. Similarly, this study found that women who received chemotherapy had a $34 \%$ increased risk of NBCM. This suggests that chemotherapy may have greater harms than benefits for many older breast cancer patients. An unexpected finding was that AET was associated with a greater than a $50 \%$ increased risk for BCSM. Over $84 \%$ of women in this study had hormone receptor positive tumors, but less than $50 \%$ received AET. Side effects of AET include accelerated boneloss, musculoskeletal pain, metabolic syndrome, vasomotor, genitourinary, mood, and sleep disturbances. [26, 27] Given these side effects, low risk of recurrence for hormone receptor positive tumors and limited life-expectancy, many older women may not initiate AET. It's plausible that the many of the older women with breast cancer who took AET were not healthy enough for other treatments or were diagnosed with later stage disease, and thus already at an increased risk for BCSM. Similarly, a study by Kimmick and colleagues (2017) observed higher rates of BCSM among women age $>70$ years who received chemotherapy, compared to younger women and adjusting for race and tumor characteristics. [14]

In addition to the increased risk of mortality that some cancer treatments pose to older patients, increased comorbidity burden and specific types of pre-existing chronic conditions were also found to increase the risk for mortality. Women with pre-existing dementia, diabetes, hypertension, 
heart disease and stroke had an increased risk for both BCSM and NBCM. Previous research has shown greater comorbidity burden from chronic conditions to increase the risk for BCSM and NBCM. [28, 29] Not only do these conditions directly increase the risk for NBCM, but they can also indirectly increase the risk of BCSM. [4, 14, 28] The presence of these conditions may increase the risk for treatment-related toxicity and complications, resulting in modified treatment regimens, or discontinuation of treatment, or omission of treatment all-together.

Unexpected findings from this study were that women who were lymph node positive and of black and Hispanic/Latina races had decreased lower risk of BCSM, compared to women who were lymph node negative or of white race. The association between race and BCSM is more difficult to interpret, but may be explained by study inclusion/exclusion criteria and adjusted hazard models controlling for receipt of specific types of treatments and GCC. It is well documented that black women with breast cancer experience poorer breast cancer survival. [30] These survival disparities are largely attributed to the greater prevalence of aggressive breast cancer subtypes diagnosed among black women and treatment disparities. This study may have limited observed survival disparities due to treatment variations by controlling for receipt of specific types of treatments, as well as, GCC in hazard models. Additionally, study criteria that excluded women enrolled in HMO plans, types of plans that are often used to manage sicker, higher cost patients, may have selected for a healthier study sample.

This study examined both BCSM and NBCM in association with a comprehensive examination of GCC, types of treatments, numerous prevalent chronic conditions, clinical, oncology resource, and demographic characteristics using a large population-based data set. Complex algorithms were used to determine receipt of GCC by calculating the correct course of care according to each patient's tumor characteristics and comparing that to the actual care received. Yet, several limitations should be kept in mind when interpreting the results of this study. This study did not measure completion of RT or chemotherapy, only the initiation of therapy. As the SEER program only began recording information about the status of the human epidermal growth factor 2 (HER2/neu) protein until breast cancer cases diagnosed in 2011, this study did not assess treatment for HER2/neu positive tumors. Nor does SEERMedicare collect information regarding results of any Oncotype testing that may influence treatment choices.

\section{Conclusion}

In conclusion, even though less than half of older women receive GCC, relatively few died from breast cancer. Preexisting chronic conditions often complicate treatment decisions and increase the risk for both BCSM and NBCM. While receipt of GCC decreases the risk of BCSM, the decision to treat should be made considering the patients existing health status, as specific types of cancer treatments, such as chemotherapy, increase the risk of NBCM. The risk and benefits of cancer treatment to the patient's health should be carefully considered before deciding the best course of treatment for each woman.

\section{Funding}

This study was partially funded by the Agency for Healthcare Research and Quality award 1 P20 HS15930-02 and the National Institute of General Medical Sciences award U54GM104942.

\section{References}

[1] Partridge AH, Hughes ME, Warner ET, Ottesen RA, Wong YN, Edge SB, et al. Subtype-Dependent Relationship Between Young Age at Diagnosis and Breast Cancer Survival. J Clin Oncol. 2016 Sep 20; 34 (27): 3308-14.

[2] Kohler BA, Sherman RL, Howlader N, Jemal A, Ryerson AB, Henry KA, et al. Annual Report to the Nation on the Status of Cancer, 1975-2011, Featuring Incidence of Breast Cancer Subtypes by Race/Ethnicity, Poverty, and State. J Natl Cancer Inst. 2015 Mar 30; 107 (6): djv048.

[3] Freedman RA, Keating NL, Lin NU, Winer EP, Vaz-Luis Lii $\mathrm{J}$, et al. Breast Cancer-Specific Survival by Age: Worse Outcomes for the Oldest Patients. Cancer. 2018 May 15; 124 (10): 2184-2191.

[4] Derks MGM, Bastiaannet E, van de Water W, de Glas, Seynaeve C, Putter H, et al. Impact of age on breast cancer mortality and competing causes of death at 10 years follow-up in the adjuvant TEAM trial. Eur J Cancer. 2018 Aug; 99: 1-8.

[5] National Comprehensive Cancer Network. Clinical practice guidelines in oncology. Available at: https://www.nccn.org/professionals/physician_gls/f_guidelines .asp\#site. Accessed August 7, 2017.

[6] Reeder-Hayes K, Peacock Hinton S, Meng K, Carey LA, Dusetzina SB. Disparities in Use of Human Epidermal Growth Hormone Receptor 2-Targeted Therapy for Early-Stage Breast Cancer. J Clin Oncol. 2016; 10; 34 (17): 2003-2009.

[7] LeMasters TJ, Madhavan SS, Sambamoorthi U, Vyas AM. Disparities in the Initial Local Treatment of Older Women with Early-Stage Breast Cancer: A Population-Based Study. J Womens Health (Larchmt). 2017; 26 (7): 735-744.

[8] LeMasters T, Madhavan S, Sambamoorthi U, Hazard H, Kelly K, Long D. Receipt of Guideline-Concordant Care among Older Women with Stage I-III Breast Cancer: A PopulationBased Study. J Natl Compr Canc Netw. 2018; 16 (6): 703-710.

[9] Inwald EC, Ortmann O, Koller M, Zeman F, Hofstadter F, Evert M, et al. Screening-relevant age threshold of 70 years and older is a stronger determinant for the choice of adjuvant treatment in breast cancer patients than tumor biology. Breast Cancer Res Treat. 2017; 163 (1): 119-130.

[10] Karavasilis V, Papadimitriou C2, Gogas H, Kouvatseas G, Pentheroudakis G, Koutras A, et al. Safety and Tolerability of Anthracycline-Containing Adjuvant Chemotherapy in Elderly High-Risk Breast Cancer Patients. Clin Breast Cancer. 2016; 16 (4): 291-298. e3. 
[11] Singh JC1, Lichtman SM. Effect of age on drug metabolism in women with breast cancer. Expert Opin Drug Metab Toxicol. 2015; 11 (5): 757-766.

[12] Sun SX, Hollenbeak CS, Leung AM. Deviation from the Standard of Care for Early Breast Cancer in the Elderly: What are the Consequences? Ann Surg Oncol. 2015; 22 (8): 24922499.

[13] Van de Water W, Bastiaannet E, Dekkers OM, de Craen AJ, Westendorp RG, Voogd AC, et al. Adherence to treatment guidelines and survival in patients with early-stage breast cancer by age at diagnosis. Br J Surg. 2012; 99 (6): 813-820.

[14] Kimmick GG, Li X, Fleming ST, Sabatino SA, Wilson JF, Lipscomb J, et al. Risk of cancer death by comorbidity severity and use of adjuvant chemotherapy among women with locoregional breast cancer. J Geriatr Oncol. 2018; 9 (3): 214-220.

[15] Berry SD, Ngo L, Samelson EJ, Kiel DP. Competing risk of death: an important consideration in studies of older adults. J Am Geriatr Soc. 2010 Apr; 58 (4): 783-787.

[16] van de Water W, Markopoulos C, van de Velde CJ, Seynaeve C, Hasenburg A, Rea D, et al. Association between age at diagnosis and disease-specific mortality among postmenopausal women with hormone receptor-positive breast cancer. JAMA. 2012; 307 (6): 579-597.

[17] Engels EA, Pfeiffer RM, Ricker W, Wheeler W, Parsons R, Warren JL. Use of Surveillance, Epidemiology, and End Results-Medicare data to conduct case-control studies of cancer among the US elderly. Am J Epidemiol. 2011; 174: 860-870.

[18] U.S. Health Resources and Services Administration, Bureau of Health Professions. Area Resource File, 2009-2010 Release Rockville, MD: U.S. Department of Health and Human Services. Fairfax, VA: Quality Resource Systems, Inc. Codebook: HE-001.

[19] Howlader N, Ries LA, Mariotto AB, Reichman ME, Ruhl J, Cronin KA. Improved estimates of cancer-specific survival rates from population-based data. J Natl Cancer Inst. 2010; 102: $1584-1598$.

[20] Carlson RW, Allred DC, Anderson BO, Burstein HJ, Carter WB, Edge SB, et al. Breast cancer. Clinical practice guidelines in oncology. J Natl Compr Canc Netw. 2009; 7 (2): 122-192.

[21] Freedman RA, Virgo KS, He Y, Pavluck AL, Winer EP, Ward
EM, Keating NL. The Association of Race/Ethnicity, Insurance Status, and Socioeconomic Factors with Breast Cancer Care. 2010; 117: 180-189.

[22] Desch C, McNiff K, Schneider E, Schrag D, McClure J, Lepisto E, et al. American Society of Clinical Oncology/National Comprehensive Cancer Network Quality Measures. J Clin Oncol. 2008; 26 (21): 3631-3637.

[23] Working Group on Health Outcomes for Older Persons with Multiple Chronic Conditions. Universal health outcome measures for older persons with multiple chronic conditions. J Am Geriatr Soc. 2012; 60: 2333-2341.

[24] Klabunde CN, Potosky AL, Legler JM, Warren JL. Development of a comorbidity index using physician claims data. J Clin Epidemiol 2000; 53 (12): 1258-67.

[25] Fine, JP and Gray, RJ. A Proportional Hazards Model for the Subdistribution of a Competing Risk. Journal of the American Statistical Association. 1999; 94: 496-509.

[26] Eastell R, Adams J, Coleman R, Howell A, Hannon RA, Cuzick J, et al. Effect of anastrozole on bone mineral density: 5 -year results from the anastrozole, tamoxifen, alone or in combination trial 18233230. J Clin Oncol. 2008; 26 (7): 10511057.

[27] Coates AS, Keshaviah A, Thürlimann B, Mouridsen H, Mauriac L, Forbes JF, et al. Five years of letrozole compared with tamoxifen as initial adjuvant therapy for postmenopausal women with endocrine-responsive early breast cancer: update of study BIG 1-98. J Clin Oncol. 2007; 25 (5): 486-92.

[28] Wollschläger D, Meng X, Wöckel A, Janni W, Kreienberg R, Blettner $\mathrm{M}$, et al. Comorbidity-dependent adherence to guidelines and survival in breast cancer-Is there a role for guideline adherence in comorbid breast cancer patients? A retrospective cohort study with 2137 patients. Breast J. 2018; 24 (2): 120-127.

[29] Klepin HD, Pitcher BN, Ballman KV, Kornblith AB, Hurria A, Winer EP, et al. Comorbidity, chemotherapy toxicity, and outcomes among older women receiving adjuvant chemotherapy for breast cancer on a clinical trial: CALGB 49907 and CALGB 361004 (alliance). J Oncol Pract. 2014; 10 (5): e285-92.

[30] Jemal A, Ward EM, Johnson CJ, Cronin KA, Ma J, Ryerson B, et al. Annual Report to the Nation on the Status of Cancer, 1975-2014, Featuring Survival. J Natl Cancer Inst. 2017; 109 (9). 